\title{
A Construction of Multisender Authentication Codes with Sequential Model from Symplectic Geometry over Finite Fields
}

\author{
Shangdi Chen ${ }^{1}$ and Chunli Yang ${ }^{2}$ \\ ${ }^{1}$ College of Science, Civil Aviation University of China, Tianjin 300300, China \\ ${ }^{2}$ Information Security Center, Beijing University of Posts and Telecommunications, P.O. Box 126, Beijing 100876, China \\ Correspondence should be addressed to Shangdi Chen; sdchen@cauc.edu.cn
}

Received 26 August 2013; Revised 26 December 2013; Accepted 5 January 2014; Published 30 April 2014

Academic Editor: Francesco Pellicano

Copyright (c) 2014 S. Chen and C. Yang. This is an open access article distributed under the Creative Commons Attribution License, which permits unrestricted use, distribution, and reproduction in any medium, provided the original work is properly cited.

Multisender authentication codes allow a group of senders to construct an authenticated message for a receiver such that the receiver can verify authenticity of the received message. In this paper, we construct multisender authentication codes with sequential model from symplectic geometry over finite fields, and the parameters and the maximum probabilities of deceptions are also calculated.

\section{Introduction}

Information security consists of confidentiality and authentication. Confidentiality is to prevent the confidential information from decrypting by adversary. The purpose of authentication is to ensure the sender is real and to verify that the information is integrated. Digital signature and authentication codes are two important means of authenticating the information and provide good service in the network. In practical, digital signature is computationally secure assuming that the computing power of adversary is limited and a mathematical problem is intractable and complex. However, authentication codes are generally safe (unconditional secure) and relatively simple. In the 1940s, C. E. Shannon first put forward the concept of perfect secrecy authentication system using the information theory. In the 1980s, information theory method had been applied to the problem of authentication by G. J. Simmons; then authentication codes became the foundation for constructing unconditionally secure authentication system. In 1974, Gilbert et al. constructed the first authentication code [1], which is a landmark in the development of authentication theory. During the same period, Simmons independently studied the authentication theory and established three participants and four participants certification models [2]. The famous mathematician Wan Zhexian constructed an authentication code without arbitration from the subspace of the classical geometry [3]. In the case of transmitter and receiver being not honest, Ma et al. constructed a series of authentication codes with arbitration [4-9]. Xing et al. constructed authentication codes using algebraic curve and nonlinear functions, respectively $[10,11]$. Safavi-Naini and Wang gave some results on multireceiver authentication codes [12]. Chen et al. made great contributions on multisender authentication codes from polynomials and matrices [13-19].

With the rapid development of information science, the traditional one-to-one authentication codes have been unable to meet the requirements of network communication, thus making the study of multiuser authentication codes particularly important. Multiuser authentication code is a generalization of traditional two-user authentication code. It can be divided into two cases: one is a sender and many receivers authentication codes; the other one is many senders and a receiver authentication codes. We call the former as multireceiver authentication codes and the latter as multisender authentication codes. Safavi-Naini R gave some results on multireceiver authentication codes using the subspace of the classical geometry, while there are only some multisender authentication codes using polynomials and matrices to construct. We present the first construction multisender authentication code using the subspace of the classical geometry, specifically symplectic geometry.

The main contribution of our paper is constructing a multi-sender authentication code using symplectic geometry. 
Furthermore, we calculate the corresponding parameters and the maximum probabilities of deceptions.

The paper is organised as follows. Section 2 gives the models of multisender authentication codes. In Section 3, we provide the calculation formulas on probability of success in attacks by malicious groups of senders. In Section 4, we give some definitions and properties on geometry of symplectic groups over finite fields. In Section 5, a construction of multisender authentication codes with sequential model from symplectic geometry over finite fields is given; then the parameters and the maximum probabilities of deceptions are also calculated. We give a comparison with the other construction of multisender authentication [19] in Section 6.

\section{Models of Multisender Authentication Codes}

We review the concepts of authentication codes which can be extracted from [20].

Definition 1 (see [20]). A systematic Cartesian authentication code $C$ is a 4 -tuple $(S, E, T ; f)$, where $S$ is the set of source states, $E$ is the set of keys, $T$ is the set of authenticators, and $f: S \times E \rightarrow T$ is the authentication mapping. The message space $M=S \times T$ is the set of all possible messages.

In the actual computer network communications, multisender authentication codes include sequential models and simultaneous models. Sequential models are that each sender uses his own encoding rules to encode a source state orderly, and the last sender sends the encoded message to the receiver; then the receiver receives the message and verifies whether the message is legal or not. Simultaneous models are that all senders use their own encoding rules to encode a source state simultaneously; then the synthesizer forms an authenticated message and sends it to the receiver; the receiver receives the message and verifies whether the message is legal or not.

In the following we will give out the working principles of two modes of multisender authentication codes and the protocols that the participants should follow.

Definition 2 (see [17]). In sequential model, there are three participants: a group of senders $U=\left\{U_{1}, U_{2}, \ldots, U_{n}\right\}$; a Key Distribution Center (KDC), for the distribution keys to senders and receiver; a receiver who receives the authenticated message and verifies the message true or not. The code works as follows: each sender and receiver has their own Cartesian authentication code, respectively. It is used to generate part of the message and verify authenticity of the received message. Sender's authentication codes are called branch authentication codes, and receiver's authentication code is called channel authentication code. Let $\left(S_{i}, E_{i}, T_{i} ; f_{i}\right)$, $i=1,2, \ldots, n$, be the $i$ th sender's Cartesian authentication codes, and let $T_{i-1} \subset S_{i}, 1 \leq i \leq n,(S, E, T ; f)$ be the receiver's Cartesian authentication code, and let $S=S_{1}, T=T_{i}, \pi_{i}$ : $E \rightarrow E_{i}$ be a subkey generation algorithm. For authenticating a message, the senders and the receiver should comply with protocols:

(1) KDC randomly selects an $e \in E$ and secretly sends it to the receiver $R$ and sends $e_{i}=\pi_{i}(e)$ to the $i$ th sender $U_{i}, i=1,2, \ldots, n$;

(2) if the senders would like to send a source state $s$ to the receiver $R, U_{1}$ calculates $t_{1}=f_{1}\left(s, e_{1}\right)$ and then sends $t_{1}$ to $U_{2}$ through an open channel; $U_{2}$ receives $t_{1}$ and calculates $t_{2}=f_{2}\left(t_{1}, e_{2}\right)$ and then sends $t_{2}$ to $U_{3}$ through an open channel. In general, $U_{i}$ receives $t_{i-1}$ and calculates $t_{i}=f_{i}\left(t_{i-1}, e_{i}\right)$ and then sends $t_{i}$ to $U_{i+1}$ through an open channel, $1<i<n . U_{n}$ receives $t_{n-1}$ and calculates $t_{n}=f_{n}\left(t_{n-1}, e_{n}\right)$ and then sends $m=\left(s, t_{n}\right)$ through an open channel to the receiver $R$;

(3) when the receiver receives the message $m=\left(s, t_{n}\right)$, he checks the authenticity by verifying whether $t_{n}=$ $f(s, e)$ or not. If the equality holds, the message is regarded as authentic and is accepted. Otherwise, the message is rejected.

Definition 3 (see [17]). In simultaneous model of a multisender authentication code, there are four participants: a group of senders $U=\left\{U_{1}, U_{2}, \ldots, U_{n}\right\}$; a Key Distribution Center (KDC), for the distribution keys to senders and receiver; a synthesizer $C$ who only runs the trusted synthesis algorithm; a receiver who receives the authenticated message and verifies the message true or not. The code works as follows: each sender and receiver has their own Cartesian authentication code, respectively. It is used to generate part of the message and verify the received message. Sender's authentication codes are called branch authentication codes, and receiver's authentication code is called channel authentication code. Let $\left(S_{i}, E_{i}, T_{i} ; f_{i}\right), i=1,2, \ldots, n$, be the sender's Cartesian authentication codes, let $(S, E, T ; f)$ be the receiver's Cartesian authentication code, let $g: T_{1} \times T_{2} \times \cdots \times$ $T_{n} \rightarrow T$ be the synthesis algorithm, and let $\pi_{i}: E \rightarrow E_{i}$ be a subkey generation algorithm. For authenticating a message, the senders and the receiver should comply with protocols:

(1) KDC randomly selects a encoding rule $e \in E$ and secretly sends it to the receiver $R$ and sends $e_{i}=\pi_{i}(e)$ to the $i$ th sender $U_{i}, i=1,2, \ldots, n$;

(2) if the senders would like to send a source state $s$ to the receiver $R, U_{i}$ computes $t_{i}=f_{i}\left(s, e_{i}\right), i=$ $1,2, \ldots, n$, and sends $m_{i}=\left(s, t_{i}\right)(i=1,2, \ldots, n)$ to the synthesizer $C$ through an open channel;

(3) the synthesizer $C$ receives the messages $m_{i}=\left(s, t_{i}\right)$, $i=1,2, \ldots, n$, and calculates $t=g\left(t_{1}, t_{2}, \ldots, t_{n}\right)$ using the synthesis algorithm $g$; then sends message $m=$ $(s, t)$ to the receiver $R$;

(4) when the receiver receives the message $m=(s, t)$, he checks the authenticity by verifying whether $t=$ $f(s, e)$ or not. If the equality holds, the message is regarded as authentic and is accepted. Otherwise, the message is rejected. 


\section{Probabilities of Deceptions}

We assume that the arbitrator (KDC) and the synthesizer (C) are credible; though they know the senders' and receiver's encoding rules, they do not participate in any communication activities. When transmitter and receiver are disputing, the arbitrator settles it. At the same time, assume that the system follows Kerckhoff's principle which the other information of the whole system is public except the actual used keys. Assume that the source state space $S$ and the receiver's decoding rules space $E_{R}$ are according to a uniform probability distribution; then the probability distribution of message space $M$ and tag space $T$ is determined by the probability distribution of $S$ and $E_{R}$. In a multisender authentication system, assume that the whole senders cooperate to form a valid message; that is, all senders as a whole and receiver are reliable. But there are some malicious senders which they together cheat the receiver; the part of senders and receiver are not credible; they can take impersonation attack and substitution attack.

Assume that $U_{1}, U_{2}, \ldots, U_{n}$ are senders, $R$ is a receiver, and $E_{i}$ is the encoding rules of $U_{i}, 1 \leq i \leq n$. $E_{R}$ is the decoding rules of receiver $R . L=\left\{i_{1}, i_{2}, \ldots, i_{l}\right\} \subset\{1,2, \ldots, n\}$, $l<n, U_{L}=\left\{U_{i_{1}}, U_{i_{2}}, \ldots, U_{i_{l}}\right\}, E_{L}=\left\{E_{i_{1}}, E_{i_{2}}, \ldots, E_{i_{l}}\right\}$.

Impersonation Attack. $U_{L}$, after receiving their secret keys, sends a message $m$ to receiver. $U_{L}$ is successful if the receiver accepts it as legitimate message. Denote $P_{I}[L]$ as the maximum probability of success of the impersonation attack. It can be expressed as

$$
P_{I}[L]=\max _{e_{L} \in E_{L}} \max _{m \in M} P\left(m \text { is accepted by } R \mid e_{L}\right) .
$$

Substitution Attack. $U_{L}$, after observing a legitimate message, substitutes it with another message $m^{\prime}$. $U_{L}$ is successful if $m^{\prime}$ is accepted by receiver as authentic. Denote $P_{S}[L]$ as the maximum probability of success of the substitution attack. It can be expressed as

$$
P_{S}[L]=\max _{e_{L} \in E_{L}} \max _{m \in M} \max _{m^{\prime} \neq m \in M} P\left(m^{\prime} \text { is accepted by } R \mid m, e_{L}\right) .
$$

\section{Symplectic Geometry}

In this section, we give some definitions and properties on geometry of symplectic groups over finite fields, which can be extracted from [20].

Let $\mathbb{F}_{q}$ be a finite field with $q$ elements, $n=2 v$ and define the $2 v \times 2 v$ alternate matrix

$$
K=\left(\begin{array}{cc}
0 & I^{(\nu)} \\
-I^{(\nu)} & 0
\end{array}\right)
$$

The symplectic group of degree $2 v$ over $\mathbb{F}_{q}$, denoted by $S p_{2 v}\left(\mathbb{F}_{q}\right)$, is defined to be the set of matrices

$$
S p_{2 v}\left(\mathbb{F}_{q}\right)=\left\{T \mid T K^{t} T=K\right\},
$$

with matrix multiplication as its group operation. Let $\mathbb{F}_{q}^{(2 v)}$ be the $2 v$-dimensional row vector space over $\mathbb{F}_{q} . S p_{2 v}\left(\mathbb{F}_{q}\right)$ has an action on $\mathbb{F}_{q}^{(2 \gamma)}$ defined as follows:

$$
\begin{gathered}
\mathbb{F}_{q}^{(2 v)} \times S p_{2 v}\left(\mathbb{F}_{q}\right) \longrightarrow \mathbb{F}_{q}^{(2 v)}, \\
\left(\left(x_{1}, x_{2}, \ldots, x_{2 v}\right), T\right) \longrightarrow\left(x_{1}, x_{2}, \ldots, x_{2 v}\right) T .
\end{gathered}
$$

The vector space $\mathbb{F}_{q}^{(2 v)}$ together with this action of $S p_{2 v}\left(\mathbb{F}_{q}\right)$ is called the symplectic space over $\mathbb{F}_{q}$.

Let $P$ be an $m$-dimensional subspace of $\mathbb{F}_{q}^{(2 v)}$. We use the same latter $P$ to denote a matrix representation of $P$; that is, $P$ is an $m \times 2 v$ matrix of rank $m$ such that its rows form a basis of $P$. The $P K^{t} P$ is alternate. Assume that it is of rank $2 s$; then $P$ is called a subspace of type $(m, s)$. It is known that subspaces of type $(m, s)$ exist in $\mathbb{F}_{q}^{(2 v)}$ if and only if

$$
2 s \leq m \leq v-s .
$$

It is also known that subspaces of the same type form an orbit under $S p_{2 v}\left(\mathbb{F}_{q}\right)$. Denote by $N(m, s ; 2 \nu)$ the number of subspaces of type $(m, s)$ in $\mathbb{F}_{q}^{(2 v)}$.

Denote by $P^{\perp}$ the set of vectors which are orthogonal to every vector of $P$; that is,

$$
P^{\perp}=\left\{y \in \mathbb{F}_{q}^{(2 v)} \mid y K^{t} x=0 \text { for all } x \in P\right\} .
$$

Obviously, $P^{\perp}$ is a $(2 v-m)$-dimensional subspace of $\mathbb{F}_{q}^{(2 v)}$.

Readers can refer to [15] for notations and terminology, which are not explained, on symplectic geometry of classical groups over finite fields.

\section{Construction}

Let $\mathbb{F}_{q}$ be a finite field with $q$ elements. Assume that $1<n<r<v \cdot U=\left\langle e_{1}, e_{2}, \ldots, e_{n}\right\rangle$; then $U^{\perp}=$ $\left\langle e_{1}, \ldots, e_{\nu}, e_{\gamma+n+1}, \ldots, e_{2 \nu}\right\rangle$. Let $W_{i}=\left\langle e_{1}, \ldots, e_{i-1}, e_{i+1}, \ldots, e_{n}\right\rangle$; then $W_{i}^{\perp}=\left\langle e_{1}, \ldots, e_{v}, e_{\nu+i}, e_{\nu+n+1}, \ldots, e_{2 \gamma}\right\rangle$. The set of source states $S=\{s \mid s$ is a subspace of type $(2 r-n, r-n)$ and $\left.U \subset s \subset U^{\perp}\right\}$; the set of $i$ th sender's encoding rules $E_{i}=\left\{e_{i} \mid e_{i}\right.$ is a subspace of type $(n+1,1), U \subset e_{i}$ and $\left.e_{i} \perp W_{i}\right\}, 1 \leq i \leq n$; the set of receiver's decoding rules $E_{R}=\left\{e_{R} \mid e_{R}\right.$ is a subspace of type $(2 n, n)$ and $\left.U \subset e_{R}\right\}$; the set of tags $T_{i}=\left\{t_{i} \mid t_{i}\right.$ is a subspace of type $(2 r-n+i, r-n+i)$ and $\left.U \subset t_{i}\right\}, 1 \leq i \leq n$.

Define the encoding maps:

$$
\begin{gathered}
f_{1}: S \times E_{1} \longrightarrow T_{1}, \quad f_{1}\left(s, e_{1}\right)=s+e_{1}, \\
f_{i}: T_{i-1} \times E_{i} \longrightarrow T_{i}, \quad f_{i}\left(t_{i-1}, e_{i}\right)=t_{i-1}+e_{i}, \quad 2 \leq i \leq n .
\end{gathered}
$$

Define the decoding map:

$$
f: S \times E_{R} \longrightarrow T_{n}, \quad f\left(s, e_{R}\right)=s+e_{R} .
$$


This code works as follows.

(1) Key Distribution. First, the KDC does a list $L$ of senders; assume that $L=\{1,2, \ldots, n\}$. Then, the KDC randomly chooses a subspace $e_{R} \in E_{R}$ and privately sends $e_{R}$ to the receiver $R$. Last, the KDC randomly chooses a subspace $e_{i} \in E_{i}$ and $e_{i} \subset e_{R}$, then privately sends $e_{i}$ to the $i$ th sender, $1 \leq i \leq n$.

(2) Broadcast. For a source state $s \in S$, the sender $U_{1}$ calculates $t_{1}=s+e_{1}$ and sends $\left(s, t_{1}\right)$ to $U_{2}$. The sender $U_{2}$ calculates $t_{2}=t_{1}+e_{2}$ and sends $\left(s, t_{2}\right)$ to $U_{3}$. Finally, the sender $U_{n}$ calculates $t_{n}=t_{n-1}+e_{n}$ and sends $m=\left(s, t_{n}\right)$ to the receiver $R$.

(3) Verification. Since the receiver $R$ holds the decoding rule $e_{R}, R$ accepts $m$ as authentic if $t_{n}=s+e_{R}$. Otherwise, it is rejected by $R$.

Lemma 4. Let $C=\left(S, E_{R}, T_{n} ; f\right), C_{1}=\left(S, E_{1}, T_{1} ; f_{1}\right), C_{i}=$ $\left(T_{i-1}, E_{i}, T_{i} ; f_{i}\right)(2 \leq i \leq n)$; then $C, C_{1}, C_{i}$ are all Cartesian authentication codes.

Proof. First, we show that $C$ is a Cartesian authentication code.

(1) For $s \in S, e_{R} \in E_{R}$. Let

$$
\begin{aligned}
& s=\left(\begin{array}{l}
U \\
Q
\end{array}\right) \begin{array}{c}
n \\
2(r-n),
\end{array} \\
& e_{R}=\left(\begin{array}{l}
U \\
V
\end{array}\right) \begin{array}{l}
n \\
n .
\end{array}
\end{aligned}
$$

From the definition of $s$ and $e_{R}$, we can assume that

$$
\begin{gathered}
\left(\begin{array}{l}
U \\
Q
\end{array}\right) K^{t}\left(\begin{array}{l}
U \\
Q
\end{array}\right)=\left(\begin{array}{ccc}
0^{(n)} & 0 & 0 \\
0 & 0 & I^{(r-n)} \\
0 & -I^{(r-n)} & 0
\end{array}\right), \\
\left(\begin{array}{l}
U \\
V
\end{array}\right) K^{t}\left(\begin{array}{l}
U \\
V
\end{array}\right)=\left(\begin{array}{cc}
0 & I^{(n)} \\
-I^{(n)} & 0
\end{array}\right) .
\end{gathered}
$$

Obviously, we have $v \notin s$ for any $v \in V$ and $v \neq 0$. Therefore,

$$
\begin{gathered}
t_{n}=s+e_{R}=\left(\begin{array}{c}
U \\
V \\
Q
\end{array}\right), \\
\left(\begin{array}{l}
U \\
V \\
Q
\end{array}\right) K\left(\begin{array}{l}
U \\
V \\
Q
\end{array}\right)=\left(\begin{array}{cccc}
0 & I^{(n)} & 0 & 0 \\
-I^{(n)} & 0 & * & * \\
0 & * & 0 & I^{(r-n)} \\
0 & * & -I^{(r-n)} & 0
\end{array}\right) .
\end{gathered}
$$

From above, $t_{n}$ is a subspace of type $(2 r, r)$ and $U \subset t_{n}$; that is, $t_{n} \in T_{n}$.

(2) For $t_{n} \in T_{n}, t_{n}$ is a subspace of type $(2 r, r)$ containing $U$. So there is subspace $V \subset t_{n}$, satisfying

$$
\left(\begin{array}{l}
U \\
V
\end{array}\right) K^{t}\left(\begin{array}{l}
U \\
V
\end{array}\right)=\left(\begin{array}{cc}
0 & I^{(n)} \\
-I^{(n)} & 0
\end{array}\right) .
$$

Then, we can assume that $t_{n}=\left(\begin{array}{c}U \\ V \\ Q\end{array}\right)$, satisfying

$$
\left(\begin{array}{l}
U \\
V \\
Q
\end{array}\right) K\left(\begin{array}{l}
U \\
V \\
Q
\end{array}\right)=\left(\begin{array}{cccc}
0 & I^{(n)} & 0 & 0 \\
-I^{(n)} & 0 & 0 & 0 \\
0 & 0 & 0 & I^{(r-n)} \\
0 & 0 & -I^{(r-n)} & 0
\end{array}\right) .
$$

Let $s=\left(\begin{array}{l}U \\ Q\end{array}\right)$; then $s$ is a subspace of type $(2 r-n, r-n)$ and $U \subset s \subset U^{\perp}$; that is, $s \in S$ is a source state. For any $v \in V$ and $v \neq 0$, we have $v \notin s$ and $V \cap U^{\perp}=\{0\}$. Therefore, $t_{n} \cap U^{\perp}=$ $\left(\begin{array}{l}U \\ Q\end{array}\right)=s$. Let $e_{R}=\left(\begin{array}{l}U \\ V\end{array}\right)$; then $e_{R}$ is a transmitter's encoding rule satisfying $t_{n}=s+e_{R}$.

If $s^{\prime}$ is another source state contained in $t_{n}$, then $U \subset s^{\prime} \subset$ $U^{\perp}$. Therefore, $s^{\prime} \subset t_{n} \cap U^{\perp}=s$, while $\operatorname{dim} s^{\prime}=\operatorname{dim} s$, so $s^{\prime}=s$. That is, $s$ is the uniquely source state contained in $t_{n}$.

Similarly, we can show that $C_{1}$ and $C_{i}(2 \leq i \leq n)$ are also Cartesian authentication code.

From Lemma 4, we know that such construction of multisender authentication codes is reasonable. Next we compute the parameters of this code.

Lemma 5. The number of the source states is $|S|=N(2(r-$ $n), r-n ; 2(\nu-n))$.

Proof. For any $s \in S$, since $U \subset s \subset U^{\perp}, s$ has the form

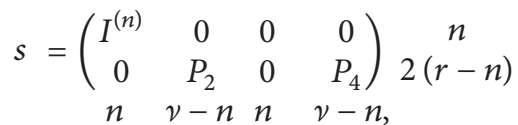

where $\left(P_{2}, P_{4}\right)$ is a subspace of type $(2(r-n), r-n)$ in the symplectic space $F_{q}^{2(\nu-n)}$. Therefore, the number of the source states is $|S|=N(2(r-n), r-n ; 2(\nu-n))$.

Lemma 6. The number of the ith sender's encoding rules is $\left|E_{i}\right|=q^{2(\nu-n)}$.

Proof. For any $e_{i} \in E_{i}, e_{i}$ is a subspace of type $(n+$ $1,1)$ containing $U$ and $e_{i}$ is orthogonal to $W_{i}$. So we can assume that $e_{i}={ }^{t}\left(e_{1}, \ldots, e_{n}, u\right)$, where $u=\left(\begin{array}{llll}x_{1} & x_{2} & \cdots & x_{2 \nu}\end{array}\right)$. Obviously, $x_{1}=\cdots=x_{n}=x_{\nu+1}=\cdots=x_{\nu+i-1}=x_{\nu+i+1}=$ $\cdots=x_{v+n}=0, x_{v+i}=1$, and $x_{n+1}, \ldots, x_{v}, x_{v+n+1}, \ldots, x_{2 v}$ arbitrarily. Therefore, $\left|E_{i}\right|=q^{2(\nu-n)}$.

Lemma 7. The number of the receiver's decoding rules is $\left|E_{R}\right|=$ $q^{2 n(\nu-n)}$

Proof. For any $e_{R} \in E_{R}$, since $e_{R}$ is a subspace of type $(2 n, n)$ containing $U, e_{R}$ has the form

$$
\begin{gathered}
e_{R}=\left(\begin{array}{cccc}
I^{(n)} & 0 & 0 & 0 \\
0 & Q_{2} & I^{(n)} & Q_{4}
\end{array}\right){ }_{n}^{n} \\
n v-n \quad n \quad v-n,
\end{gathered}
$$

where $Q_{2}, Q_{4}$ are arbitrary matrices. Therefore, $\left|E_{R}\right|=$ $q^{2 n(\nu-n)}$ 
Lemma 8. (1) The number of decoding rules $e_{R}$ contained in $t_{n}$ is $q^{2 n(r-n)}$;

(2) the number of the tags is $\left|T_{n}\right|=q^{2 n(v-r)} N(2(r-n), r-$ $n ; 2(\nu-n))$.

Proof. (1) For any $t_{n} \in T_{n}, t_{n}$ is a subspace of type $(2 r, r)$ and $U \subset t_{n}$. We assume that $t_{n}$ has the form

$$
\begin{aligned}
& t_{n} \\
& =\left(\begin{array}{cccccc}
I^{(n)} & 0 & 0 & 0 & 0 & 0 \\
0 & I^{(r-n)} & 0 & 0 & 0 & 0 \\
0 & 0 & 0 & I^{(n)} & 0 & 0 \\
0 & 0 & 0 & 0 & I^{(r-n)} & 0 \\
n & r-n & v-r & n & r-n & \nu-r . \\
n & r-n \\
& & & & &
\end{array}\right.
\end{aligned}
$$

If $e_{R} \subset t_{n}$, then we can assume that

$$
e_{R}=\left(\begin{array}{cccccc}
I^{(n)} & 0 & 0 & 0 & 0 & 0 \\
0 & R_{2} & 0 & I^{(n)} & R_{5} & 0
\end{array}\right) n
$$

where $R_{2}, R_{5}$ are arbitrary matrices. Therefore, the number of $e_{R}$ contained in $t_{n}$ is $q^{2 n(r-n)}$.

(2) We know that a tag contains only one source state and the number of decoding rules $e_{R}$ contained in $t_{n}$ is $q^{2 n(r-n)}$. Therefore, we have $\left|T_{n}\right|=|S|\left|E_{R}\right| / q^{2 n(r-n)}=q^{2 n(\nu-r)} N(2(r-$ $n), r-n ; 2(\nu-n))$.

Theorem 9. The parameters of the above constructed multisender authentication code are

$$
\begin{aligned}
& |S|=N(2(r-n), r-n ; 2(\nu-n)) ; \\
& \left|E_{i}\right|=q^{2(\nu-n)} ; \\
& \left|E_{R}\right|=q^{2 n(\nu-n)} ; \\
& \left|T_{n}\right|=q^{2 n(\nu-r)} N(2(r-n), r-n ; 2(\nu-n)) .
\end{aligned}
$$

Without loss of generality, we can assume that $U_{L}=$ $\left\{U_{1}, U_{2}, \ldots, U_{l}\right\}, E_{L}=\left\{E_{1} \times \cdots \times E_{l}\right\}$, where $l<n$.

Lemma 10. For any $e_{L}=\left(e_{1}, e_{2}, \ldots, e_{l}\right) \in E_{L}$, the number of $e_{R}$ containing $e_{L}$ is $q^{2(n-l)(\nu-n)}$.

Proof. For any $e_{L}=\left(e_{1}, e_{2}, \ldots, e_{l}\right) \in E_{L}$, we can assume that

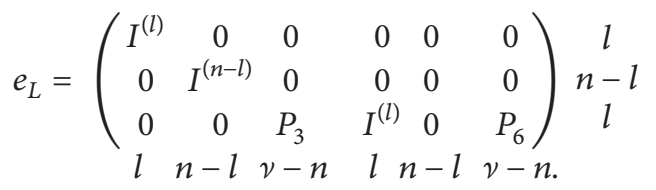

If $e_{L} \subset e_{R}$, then $e_{R}$ has the form

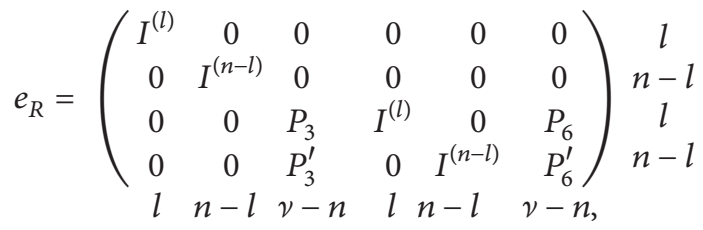

where $P_{3}^{\prime}, P_{6}^{\prime}$ are arbitrary matrices. Therefore, the number of $e_{R}$ containing $e_{L}$ is $q^{2(n-l)(\nu-n)}$.

Lemma 11. For any $t_{n} \in T_{n}$ and $e_{L}=\left(e_{1}, e_{2}, \ldots, e_{l}\right) \in E_{L}$, the number of $e_{R}$ contained in $t_{n}$ and containing $e_{L}$ is $q^{2(n-l)(r-n)}$.

Proof. For any $t_{n} \in T_{n}, t_{n}$ is a subspace of type $(2 r, r)$ and $U \subset t_{n}$. We assume that $t_{n}$ has the form

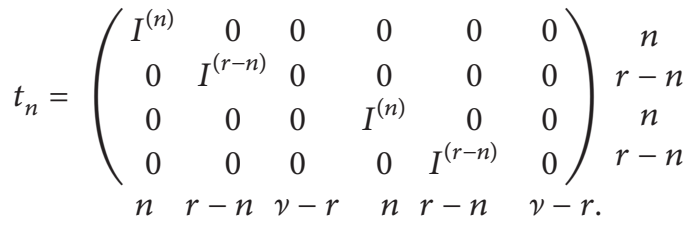

If $e_{L} \subset t_{n}$, assume that $e_{L}$ has the form

$$
e_{L}=\left(\begin{array}{cccccccc}
I^{(l)} & 0 & 0 & 0 & 0 & 0 & 0 & 0 \\
0 & I^{(n-l)} & 0 & 0 & 0 & 0 & 0 & 0 \\
0 & 0 & R_{3} & 0 & I^{(l)} & 0 & R_{7} & 0
\end{array}\right) \begin{gathered}
l \\
l
\end{gathered}
$$

If $e_{R} \subset t_{n}$ and $e_{L} \subset e_{R}$, then

$$
e_{R}=\left(\begin{array}{cccccccc}
I^{(l)} & 0 & 0 & 0 & 0 & 0 & 0 & 0 \\
0 & I^{(n-l)} & 0 & 0 & 0 & 0 & 0 & 0 \\
0 & 0 & R_{3} & 0 & I^{(l)} & 0 & R_{7} & 0 \\
0 & 0 & R_{3}^{\prime} & 0 & 0 & I^{(n-l)} & R_{7}^{\prime} & 0
\end{array}\right) \begin{gathered}
l \\
n-l \\
l \\
l
\end{gathered}
$$


where $R_{3}^{\prime}, R_{7}^{\prime}$ are arbitrary matrices. Therefore, the number of $e_{R}$ contained in $t_{n}$ and containing $e_{L}$ is $q^{2(n-l)(r-n)}$.

Lemma 12. Assume that $t_{n} \in T_{n}$ and $t_{n}^{\prime} \in T_{n}$ are two distinct tags which are decoded by receiver's decoding rule $e_{R} . s_{1}$ and $s_{2}$ contained in $t_{n}$ and $t_{n}^{\prime}$, respectively. Let $s_{0}=s_{1} \cap s_{2}$, dim $s_{0}=k$; then $n \leq k \leq 2 r-n-1$; the number of $e_{R}$ contained in $t_{n} \cap t_{n}^{\prime}$ and containing $e_{L}$ is $q^{(n-l)(k-n)}$.

Proof. Since $t_{n}=s_{1}+e_{R}, t_{n}^{\prime}=s_{2}+e_{R}$ and $t_{n} \neq t_{n}^{\prime}$, then $s_{1} \neq s_{2}$. And for any $s \in S, U \subset s$, therefore, $n \leq k \leq 2 r-n-1$. Assume that $s_{i}^{\prime}$ is the complementary subspace of $s_{0}$ in the $s_{i}$; then $s_{i}=s_{0}+s_{i}^{\prime}(i=1,2)$. Because of $t_{n}=s_{1}+e_{R}=s_{0}+s_{1}^{\prime}+e_{R}$, $t_{n}^{\prime}=s_{2}+e_{R}=s_{0}+s_{2}^{\prime}+e_{R}$ and $s_{1}=t_{n} \cap U^{\perp}, s_{2}=t_{n}^{\prime} \cap U^{\perp}$, we know $s_{0}=\left(t_{n} \cap U^{\perp}\right) \cap\left(t_{n}^{\prime} \cap U^{\perp}\right)=t_{n} \cap t_{n}^{\prime} \cap U^{\perp}=s_{1} \cap t_{n}^{\prime}=s_{2} \cap t_{n}$, and $t_{n} \cap t_{n}^{\prime}=\left(s_{1}+e_{R}\right) \cap t_{n}^{\prime}=\left(s_{0}+s_{1}^{\prime}+e_{R}\right) \cap t_{n}^{\prime}=\left(\left(s_{0}+e_{R}\right)+s_{1}^{\prime}\right) \cap t_{n}^{\prime}$. Since $s_{0}+e_{R} \subseteq t_{n}^{\prime}$, then $t_{n} \cap t_{n}^{\prime}=\left(s_{0}+e_{R}\right)+\left(s_{1}^{\prime} \cap t_{n}^{\prime}\right)$, while $s_{1}^{\prime} \cap t_{n}^{\prime} \subseteq s_{1} \cap t_{n}^{\prime}=s_{0}$, so $t_{n} \cap t_{n}^{\prime}=s_{0}+e_{R}$.

From the definition of the $t_{n}$ and $t_{n}^{\prime}$, we assume that

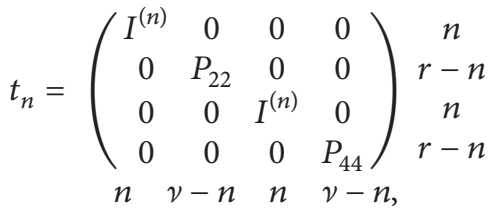

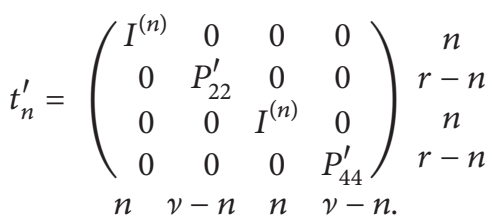

Let

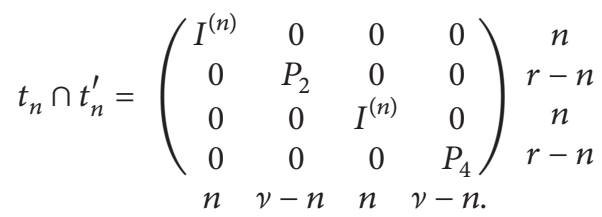

And from above we know that $t_{n} \cap t_{n}^{\prime}=s_{0}+e_{R}$; then $\operatorname{dim}\left(t_{n} \cap\right.$ $\left.t_{n}^{\prime}\right)=k+n$; therefore,

$$
\operatorname{dim}\left(\begin{array}{cccc}
0 & P_{2} & 0 & 0 \\
0 & 0 & 0 & P_{4}
\end{array}\right)=k-n
$$

For any $e_{L} \subset t_{n} \cap t_{n}^{\prime}$, we assume that

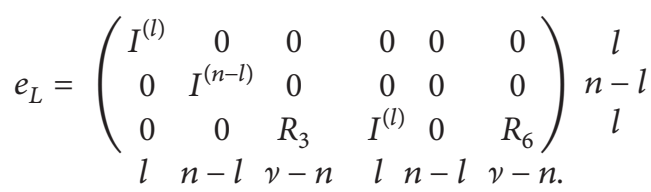

If $e_{R} \subset t_{n} \cap t_{n}^{\prime}$ and $e_{L} \subset e_{R}$, then $e_{R}$ has the form

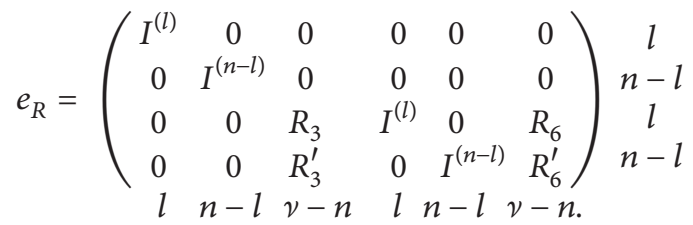

So, every row of $\left(\begin{array}{llll}0 & R_{3}^{\prime} & 0 & R_{6}^{\prime}\end{array}\right)$ is the linear combination of $\left(\begin{array}{cccc}0 & P_{2} & 0 & 0 \\ 0 & 0 & 0 & P_{4}\end{array}\right)$. Therefore, the number of $e_{R}$ contained in $t_{n} \cap t_{n}^{\prime}$ and containing $e_{L}$ is $q^{(n-l)(k-n)}$.

Theorem 13. In the constructed multi-sender authentication codes, the maximum probabilities of success for impersonation attack and substitution attack from $U_{L}$ on the receiver $R$ are

$$
P_{I}(L)=\frac{1}{q^{2(n-l)(\nu-r)}}, \quad P_{S}(L)=\frac{1}{q^{(n-l)}} .
$$

Proof. (1) Impersonation Attack. $U_{L}$, after receiving their secret keys, sends a message $m$ to $R$. $U_{L}$ is successful if the receiver accepts it as authentic. Therefore,

$$
\begin{aligned}
P_{I}(L) & =\max _{e_{L} \in E_{L}} \max _{m \in M}\left\{\frac{\left|\left\{e_{R} \in E_{R} \mid e_{L} \subset e_{R}, e_{R} \subset t\right\}\right|}{\left|\left\{e_{R} \in E_{R} \mid e_{L} \subset e_{R}\right\}\right|}\right\} \\
& =\frac{q^{2(n-l)(r-n)}}{q^{2(n-l)(v-n)}} \\
& =\frac{1}{q^{2(n-l)(v-r)}} .
\end{aligned}
$$

(2) Substitution Attack. $R_{L}$, after observing a message $m$ that is transmitted by the sender, replaces $m$ with another message $m^{\prime} . R_{L}$ is successful if $m^{\prime}$ is accepted by $R$ as authentic. Therefore,

$P_{S}(L)$

$=\max _{e_{L} \in E_{L}} \max _{m \in M_{m \neq m^{\prime} \in M}} \max _{m \neq}\left\{\frac{\left|\left\{e_{R} \in E_{R} \mid e_{L} \subset e_{R}, e_{R} \subset t, e_{R} \subset t^{\prime}\right\}\right|}{\left|\left\{e_{R} \in E_{R} \mid e_{L} \subset e_{R}, e_{R} \subset t\right\}\right|}\right\}$

$=\max _{n \leq k \leq 2 r-n-1} \frac{q^{(n-l)(k-n)}}{q^{2(n-l)(r-n)}}$

$=\frac{1}{q^{(n-l)}}$.

\section{The Advantage of the Constructed Authentication Code}

The security of an authentication code could be measured by the maximum probabilities of deceptions. The smaller the probability of successful attack, the higher the security of the authentication codes. Now let us compare the security of our constructed authentication code with the known one [19].

The constructed authentication code in [19] is also a multisender authentication code from symplectic geometry over finite fields, but which is in simultaneous model. If we choose the parameters $n, n^{\prime}, r$, and $v$ with $1<n<$ $n^{\prime}<r<v, n>(r / 2)$, and $n^{\prime}-n>v-r$, from Table 1 we see that the maximum probabilities of deceptions of our construction are smaller than the construction in [19]. Therefore, compared with the construction in [19], our construction is more efficient. 
TABLE 1: $\left(n>r / 2, n^{\prime}-n>v-r\right)$.

\begin{tabular}{|c|c|c|c|}
\hline Constructions & {$[19]$} & Size relation & Ours \\
\hline The number of senders & $n$ & $=$ & $n$ \\
\hline The number of attackers & $l, 1 \leq l<n$ & $=$ & $l, 1 \leq l<n$ \\
\hline \multicolumn{4}{|l|}{ The parameters of codes } \\
\hline$|S|$ & $N(2(r-n), r-n ; 2(\nu-n))$ & $=$ & $N(2(r-n), r-n ; 2(\nu-n))$ \\
\hline$\left|E_{i}\right|$ & $q^{2(\nu-n)}$ & $=$ & $q^{2(\nu-n)}$ \\
\hline$\left|E_{R}\right|$ & $q^{2 n^{\prime}\left(\nu-n^{\prime}\right)}$ & $>$ & $q^{2 n(\nu-n)}$ \\
\hline$|T|$ & $N(2(r-n), r-n ; 2(\nu-n)) q^{2 n^{\prime}\left(\nu-r-n^{\prime}+n\right)}$ & $<$ & $N(2(r-n), r-n ; 2(\nu-n)) q^{2 n(\nu-r)}$ \\
\hline \multicolumn{4}{|c|}{ The probabilities of deceptions } \\
\hline$P_{I}(L)$ & 1 & & 1 \\
\hline$P_{S}(L)$ & $\begin{array}{l}q^{2\left(n^{\prime}-l\right)\left(v+n-n^{\prime}-r\right)-\left(n^{\prime}-n\right)(n-l)} \\
\frac{1}{\left(^{\prime}-l\right)\left(2 n-2 n^{\prime}+1\right)+\left(n^{\prime}-n\right)(n-l)}\end{array}$ & $>$ & $\begin{array}{c}\overline{q^{2(n-l)(v-r)}} \\
\frac{1}{a^{n-l}}\end{array}$ \\
\hline
\end{tabular}

\section{Conflict of Interests}

The authors declare that there is no conflict of interests regarding the publication of this paper.

\section{Acknowledgments}

The Project is sponsored by the National Natural Science Foundation of China (no. 61179026) and the Fundamental Research Funds of the Central Universities (no. 3122013 K001).

\section{References}

[1] E. N. Gilbert, F. J. MacWilliams, and N. J. A. Sloane, "Codes which detect deception," The Bell System Technical Journal, vol. 53, pp. 405-424, 1974.

[2] G. J. Simmons, "Message authentication with arbitration of transmitter/receiver disputes," in Proceedings of the 6th Annual International Conference on Theory and Application of Cryptographic Techniques (Eurocrypt '87), vol. 304, pp. 151-165, 1988.

[3] Z. X. Wan, "Construction of cartesian authentication codes from unitary geometry," Designs, Codes and Cryptography, vol. 2, no. 4, pp. 333-356, 1992.

[4] W. P. Ma and X. M. Wang, "A construction of authentication codes with arbitration based on symplectic spaces," Chinese Journal of Computers, vol. 22, no. 9, pp. 949-952, 1999.

[5] G. You, S. Xinhua, and W. Hongli, "Construction of authentication codes with arbitration from symplectic geometry over finite fields," Acta Scientiarum Naturalium Universitatis Nankaiensis, vol. 41, no. 6, pp. 72-77, 2008.

[6] S. Chen and D. Zhao, "New construction of authentication codes with arbitration from pseudo-symplectic geometry over finite fields," Ars Combinatoria, vol. 97, pp. 453-465, 2010.

[7] S. Chen and D. Zhao, "Two constructions of optimal Cartesian authentication codes from unitary geometry over finite fields," Acta Mathematicae Applicatae Sinica, vol. 29, no. 4, pp. 829-836, 2013.

[8] S. Chen and D. Zhao, "Construction of multi-receiver multifold authentication codes from singular symplectic geometry over finite fields," Algebra Colloquium, vol. 20, no. 4, pp. 701710, 2013.
[9] L. Ruihu and L. Zunxian, "Construction of $A^{2}$-codes from symplectic geometry," Journal of Shanxi Normal University, vol. 26, no. 4, pp. 10-15, 1998.

[10] C. Xing, H. Wang, and K.-Y. Lam, "Constructions of authentication codes from algebraic curves over finite fields," IEEE Transactions on Information Theory, vol. 46, no. 3, pp. 886-892, 2000.

[11] C. Carlet, C. Ding, and H. Niederreiter, "Authentication schemes from highly nonlinear functions," Designs, Codes and Cryptography, vol. 40, no. 1, pp. 71-79, 2006.

[12] R. Safavi-Naini and H. Wang, "Multireceiver authentication codes: models, bounds, constructions, and extensions," Information and Computation, vol. 151, no. 1-2, pp. 148-172, 1999.

[13] S. Chen and D. Zhao, "Construction of multi-receiver multifold authentication codes from singular symplectic geometry over finite fields," Algebra Colloquium, vol. 20, no. 4, pp. 701710, 2013.

[14] S. Chen and D. Zhao, "Two constructions of multireceiver authentication codes from symplectic geometry over finite fields," Ars Combinatoria, vol. 99, pp. 193-203, 2011.

[15] Y. Desmedt, Y. Frankel, and M. Yung, "Multi-receiver/multisender network security: efficient authenticated multicast/feedback," in Proceedings of the the 11th Annual Conference of the IEEE Computer and Communications Societies, vol. 3, pp. 2045-2054, May 1992.

[16] Q. Yingchun and Z. Tong, "Multiple authentication Code with multi-transmitter and its constructions," Journal of Zhongzhou University, vol. 20, no. 1, pp. 118-120, 2003.

[17] M. Wen-Ping and W. Xin-Mei, "Several new constructions on multitransmitters authentication codes," Acta Electronica Sinica, vol. 28, no. 4, pp. 117-119, 2000.

[18] D. Qingling and L. Shuwang, "Bounds and construction for multi-sender authentication code," Computer Engineering and Applications, vol. 10, pp. 9-10, 2004.

[19] S. Chen and C. Yang, "A new construction of multisender authentication codes from symplectic geometry over finite fields," Ars Combinatoria, vol. 106, pp. 353-366, 2012.

[20] Z. Wan, Geometry of Classical Groups over Finite Fields, Science Press, Beijing, China, 2nd edition, 2002. 


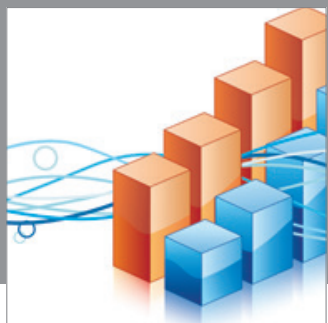

Advances in

Operations Research

mansans

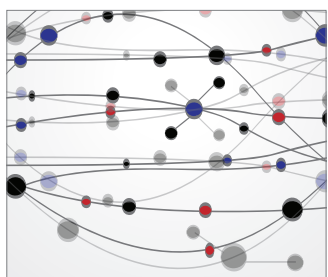

The Scientific World Journal
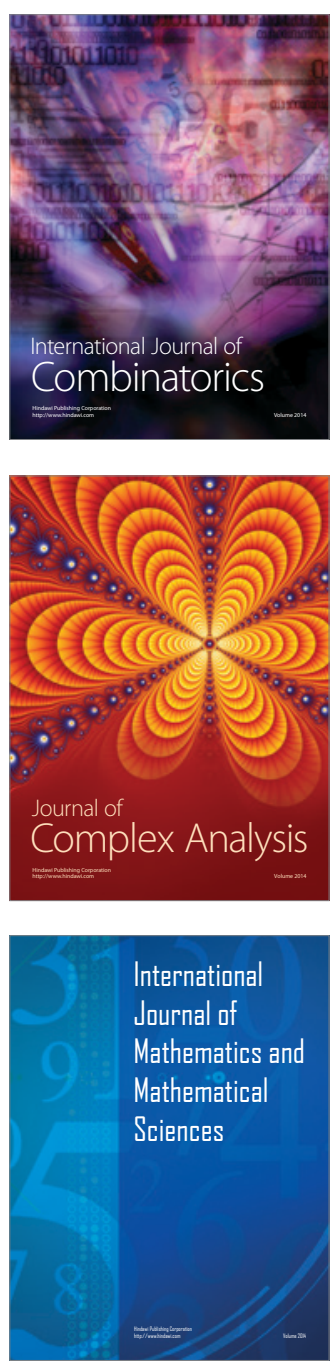
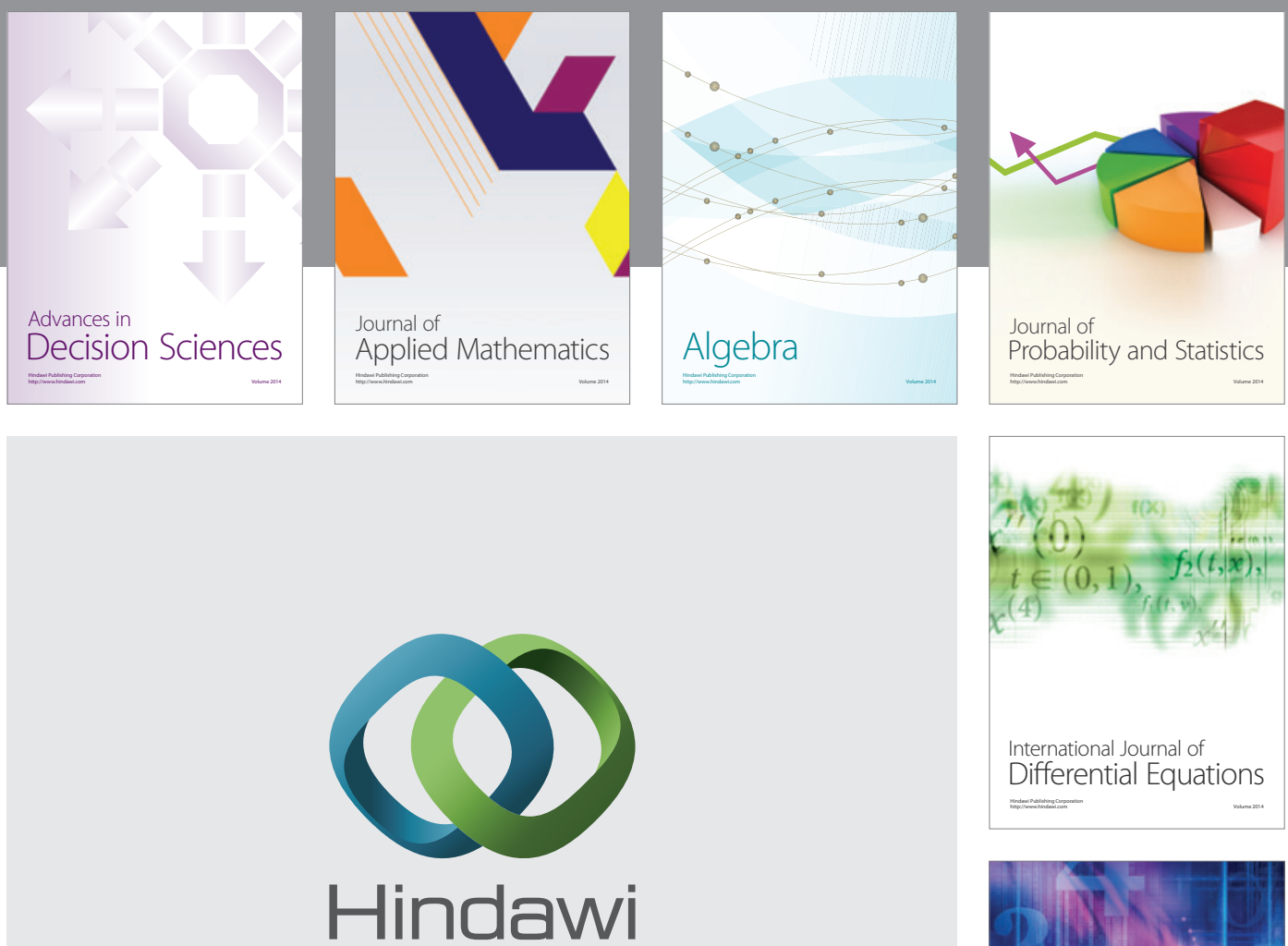

Submit your manuscripts at http://www.hindawi.com
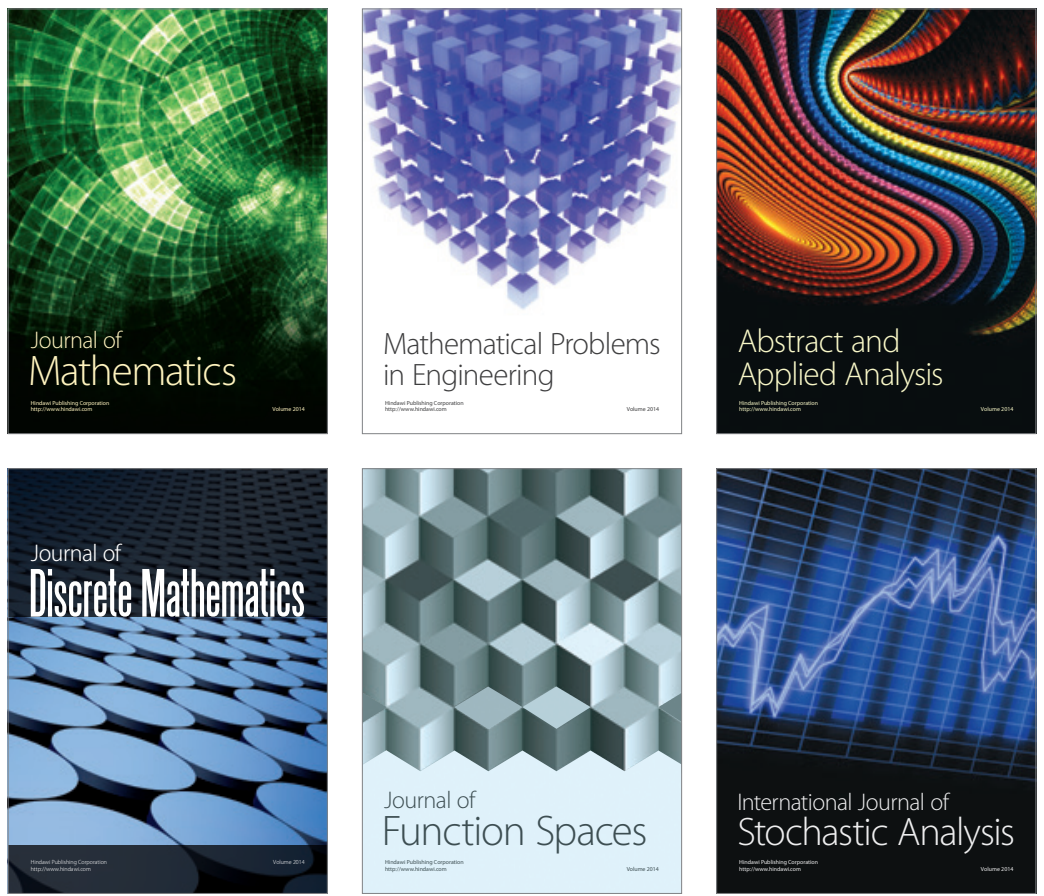

Journal of

Function Spaces

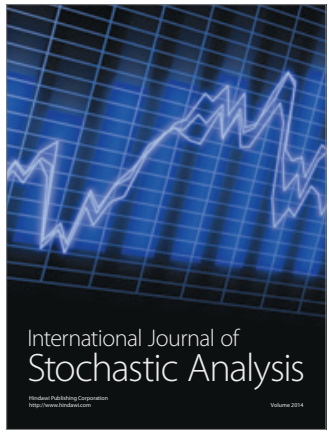

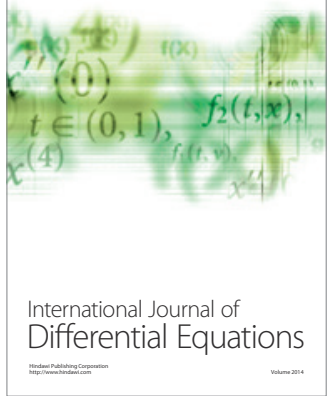
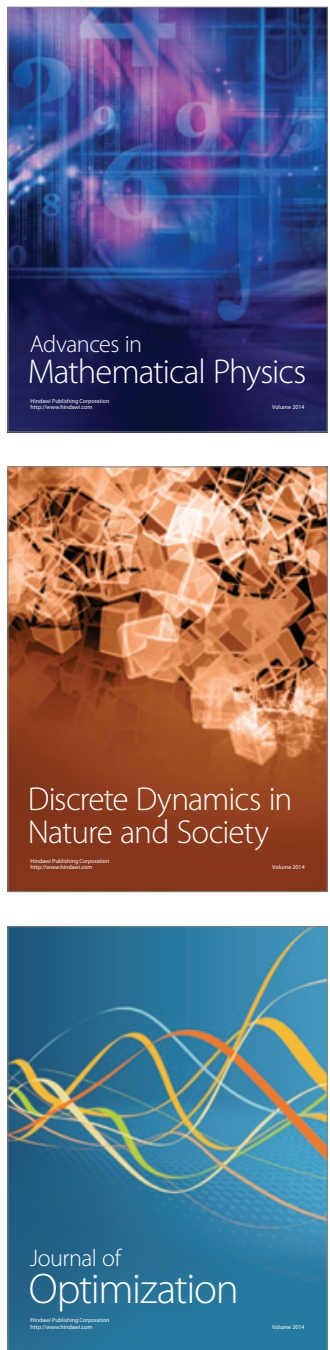